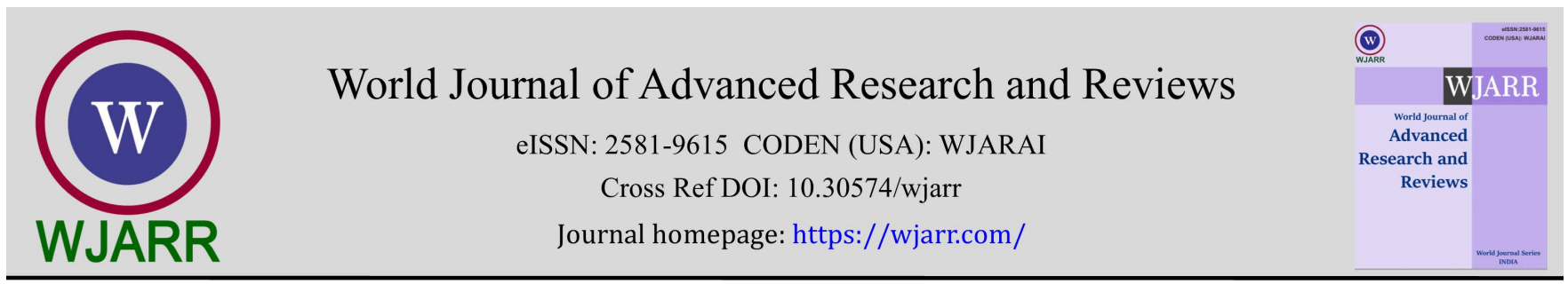

(REVIEW ARTICLE)

\title{
Liposome assisted drug delivery-A review
}

\author{
Joseph Johny*, Sinitha Sreedhar, Aiswarya P R, Athulya B Mohan and Kavya A S \\ Department of Oral Medicine and Radiology Sree Anjaneya Institute of Dental Sciences Modakkallur, Atholi, Calicut.
}

World Journal of Advanced Research and Reviews, 2021, 12(03), 594-601

Publication history: Received on 22 November 2021; revised on 28 December 2021; accepted on 30 December 2021

Article DOI: https://doi.org/10.30574/wjarr.2021.12.3.0739

\begin{abstract}
Liposomes, sphere-formed vesicles consisting of one or greater phospholipid bilayers, had been first described within the mid-60s. Among numerous gifted new drug delivery systems, liposomes signify an advanced generation to supply active molecules to the site of action, and right now, numerous formulations are in clinical use.

The application of liposomes to help drug shipping has already had a chief impact on many biomedical regions. They have been proven to be beneficial for stabilizing pharmaceuticals, overcoming boundaries to cellular and tissue uptake, and improving biodistribution of compounds to goal sites In vivo. This permits powerful delivery of encapsulated compounds to goal sites even as minimizing systemic toxicity. Liposomes present as an attractive transport gadget due to their wide physicochemical and biophysical properties which allow smooth manipulation to cope with exclusive shipping concerns. In this review, we will talk the advances in liposome assisted drug shipping, biological challenges, and present day medical and experimental use of liposomes for biomedical applications. The translational limitations of liposomal technology may also be provided.
\end{abstract}

Keywords: Liposome; Drug delivery; Reticuloendothelial system; Opsonisation

\section{Introduction}

Liposomes are small synthetic vesicles of spherical form that may be comprised of cholesterol and natural nonpoisonous phospholipids. Liposomes are appreciably used as companies for several molecules in cosmetic and pharmaceutical industries. Liposomes were the primary nanoscale drug to be authorized for scientific use in 1995 . First being described through English hematologist Alec Bangham in 1961. Liposomes are the most common place and wellinvestigated nanocarriers for focused drug shipping.

Liposomes are defined as phospholipid vesicles consisting of one or extra concentric lipid bilayers enclosing discrete aqueous areas. Artificial lipid vesicles (additionally called liposomes) were diagnosed and notably used as transport vehicles for pharmaceuticals. The phospholipid bilayer envelope is a cell-like boundary appropriate for cell investigations and presents liposomes a useful scaffold appropriate for essential cellular features which includes motility and form.

Liposomal encapsulation technology (LET) is the most up-to-date shipping method utilized by scientific investigators to transmit tablets that act as healing promoters to the assured frame organs. Liposomes form a barrier around their contents, which is proof against enzymes within the mouth and stomach, alkaline solutions, digestive juices, bile salts, and intestinal flora that are generated inside the human frame, as well as free radicals. The contents of the liposomes are, consequently, covered from oxidation and degradation.

\footnotetext{
${ }^{*}$ Corresponding author: Joseph Johny

Department of Oral Medicine and Radiology Sree Anjaneya Institute of Dental Sciences Modakkallur, Atholi,Calicut.

Copyright $(2021$ Author(s) retain the copyright of this article. This article is published under the terms of the Creative Commons Attribution Liscense 4.0.
} 
This shielding phospholipid defend or barrier remains undamaged until the contents of the liposome are added to the precise goal gland, organ, or system in which the contents might be applied [1].

The precise capacity of liposomal structures to entrap each lipophilic and hydrophilic compounds allows a various variety of drugs to be encapsulated via these vesicles. Hydrophobic molecules are inserted into the bilayer membrane, and hydrophilic molecules may be entrapped within the aqueous center [2]. Liposomal formulations are characterised by particle size, charge, quantity of lamellae, lipid composition, and surface modification with polymers and ligandsthose all govern their stability In vitro and In vivo [3]. Encapsulation within liposomes protects compounds from early inactivation, degradation and dilution inside the move [4]. Liposomes are commonly taken into consideration to be pharmacologically inactive with minimum toxicity, as they have a tendency to be composed of natural phospholipids [5] but growing variety of studies have shown that liposomes are not as immunologically inert as soon as suggested [6] . This assessment will deal with the advances, biological challenges, biomedical applications, and translational barriers of liposomal technology.

\section{Liposomal drug delivery platforms}

There are four key varieties of liposomal transport systems-conventional liposomes, sterically-stabilized liposomes, ligand-targeted liposomes, and a combination of the above. Conventional liposomes were the first generation of liposomes to be advanced. They include a lipid bilayer that may be composed of cationic, anionic, or neutral (phospho)lipids and cholesterol, which encloses an aqueous quantity research at the clinical capacity of traditional liposomes commenced within the Eighties, wherein liposomal delivery proved useful for improving the healing index of encapsulated capsules, consisting of doxorubicin and amphotericin [7].conventional liposomal formulations decreased the toxicity of compounds In vivo, thru enhancing pharmacokinetics and biodistribution to elicit drug delivery to diseased tissue in evaluation to unfastened drug. But the shipping system was susceptible to rapid elimination from the bloodstream, consequently restricting its healing efficacy [8]. This rapid clearance due to opsonization of plasma components and uptake by using constant macrophages of the reticuloendothelial system (RES), mainly within the liver and spleen [9]

To enhance liposome balance and improve their circulation times in the blood, sterically-stabilized liposomes have been delivered. The hydrophilic polymer, polyethylene glycol (PEG), has been shown to be the most advantageous desire for acquiring sterically-stabilized liposomes.

The established order of a steric barrier improves the efficacy of encapsulated agents by way of lowering In vivo opsonization with serum additives, and the fast popularity and uptake by the RES. This not handiest reduces the elimination of medication via prolonging blood stream and providing accumulation at pathological sites, however additionally attenuates side effects[10] Steric stabilization strongly influences the pharmacokinetics of liposomes [11] . At the same time as coating liposomes with PEG effects in extended movement instances, there may be an offsetting reduction within the capability to have interaction with the supposed targets [12]

Ligand-targeted liposomes offer a huge capacity for site-precise transport of medicine to particular cells or organs In vivo, which selectively express or over express particular ligands (e.g., receptors or mobile adhesion molecules) at the site of disease [13]. Many kinds of ligands are available consisting of antibodies, peptides/proteins and carbohydrates. The coupling of antibodies, in particular monoclonal antibodies, to create immunoliposomes represents one of the more flexible ligands that may be affixed to liposome surfaces [14]. One of the advantages of the use of monoclonal antibodies is theirs ability and higher binding avidity due to the presence of two binding sites on the molecule.

\section{Liposome in clinical use}

For the reason that first liposomal gadget for drug shipping, Doxil®, turned into authorized in 1995, there were many technological improvements in liposomal transport structures which have brought about delivery structures with vastly advanced physicochemical properties. The 3 guiding standards that have been actual at the time of Doxilß’s approval, but, ring real these days as nicely and can be carried out to all liposomal transport structures regardless of type. Liposomal drug delivery structures promise (i) prolonged drug flow time and avoidance of the reticuloendothelial system due to using PEGylated nano-liposomes; (ii) high and stable remote loading of the encapsulate drug; and (iii) optimizing the composition of the phospholipid membrane to obtain improved balance. Many liposomal modifications are on the market with greater in clinical development. A number of the most a hit transport methods depend on PEG conjugated lipids. In truth, the primary FDA accredited nano-drug, doxorubicin, is added the usage of PEGylated liposomes [15] regularly utilized in aggregate with different drugs, PLD treats various varieties of cancer such as AIDS- 
related Kaposi's sarcoma, leukemia, and ovarian, breast, bone, lung, and brain cancers. PLD has also been determined to be an effective opportunity to standard doxorubicin inpatients with pre present cardiac disorder [16]. When doxorubicin is integrated in PEGylated liposomes, it minimizes the uptake and clearance with the aid of the RES, which prolongs the serum and plasma -existence. This permits the PLD to accumulate inside the tumor tissue, as opposed to in non-target healthy tissues [17]. Every other kind of PEGylated liposome presently in phase I trials is PEPO2, an irinotecan-encapsulated liposomal components used to treat advanced refractory stable tumors [18]. Camptothecin, which is formulated in PEGylated stealth liposomes, is likewise in phase I trials for ovarian cancers remedy [19].

\section{Biological Challenges Facing Liposomal Drug Delivery Systems}

As with any foreign particle that enters the body, liposomes come upon multiple defense structures aimed toward identification, neutralization, and removal of invading materials. Those defenses include the RES, opsonization, and immunogenicity [20]. Even as these limitations must be circumvented for most desirable liposome feature, different factors such as the enhanced permeability and retention (EPR) impact may be exploited to enhance drug shipping [21].

\section{The Reticuloendothelial System (RES) and Liposome Clearance}

The RES is the principle site of liposome accumulation following their systemic administration [22]. The main organs related to the RES include the liver, spleen, kidney, lungs, bone marrow, and lymph nodes [23]. The capacity of the RES to sequester liposomes from the blood stream is attributed to fenestrations in their microvasculature. Pore diameters in those capillaries can range from one 100 to $800 \mathrm{~nm}$, that is big sufficient for the extravasation and removal of most drug-loaded liposomes (50-1000 $\mathrm{nm}$ in size) [24].Liposomes are cleared inside the RES through resident macrophages through direct interactions with the phagocytic cells [25]. Conjugation of PEG polymers to the liposomal membrane is a key approach for improving movement times and stopping elimination with the aid of the RES through steric stabilization [26]. PEGylation creates a nearby surface concentration of tremendously hydrated companies which sterically inhibits each electrostatic and hydrophobic reactions with plasma proteins and/or cells and thereby reduces liposomal uptake by using the RES [27].

\section{Opsonins and Vesicle Destabilization}

The degree of interplay between liposomal drug delivery structures and plasma proteins is important in figuring out overall nanocarrier biodistribution, efficacy, and toxicity [28]. Plasma proteins had been shown to play a pivotal position in liposomal clearance with the aid of the RES through opsonization, in addition to in vesicular destabilization [29]. Opsonization of liposomes by way of serum proteins depends on a variety of things including size, surface charge and stability [30]. The volume of this interplay has been proven to lower with liposome size from 800 to $200 \mathrm{~nm}$ in diameter, as small liposomes can't assist opsonic activity [31]. The inclusion of cholesterol is an important element for growing liposome stability and minimizing phospholipid exchange [32]. Incorporation of cholesterol into the liposomal membrane abates lipid exchange with other circulating systems (e.g., red blood cells and lipoproteins) that may cause the depletion of excessive high phase transition temperature lipids and their substitute with much less physiologically solid additives [33]. Integrating cholesterol into small (about $100 \mathrm{~nm}$ ), electrostatically neutral liposomes has been proven to lengthen circulate time inside the range of numerous hours [34].

\section{The Accelerated Blood Clearance (ABC) Phenomenon}

The interaction of liposome components with the immune system has contributed to the challenges in translation to medical use synthetic adjustments to improve their application as drug delivery vehicles can bring about antibody production towards their numerous additives and/or the encapsulated product.As an instance, repeated injection of PEGylated liposomes has been related to loss of their long circulating properties and next clearance from the blood [35]. This phenomenon is known as the "Accelerated blood clearance" (ABC) phenomenon. The ABC phenomenon is a major problem for the scientific application of PEGylated formulations that require multiple dosing regimens. Management of higher doses of the initial PEGylated liposomes ( $>1 \mu \mathrm{mol}$ phospholipids $/ \mathrm{kg}$ ) have been shown to reduce the significance of the ABC phenomenon [36] Enhancing the phospholipid dose has shown PEG-reactive B cells to turn out to be apoptotic lowering anti-PEG IgM production and as a result abating the ABC phenomenon [37]. The ABC phenomenon has no longer been stated to occur in patients receiving PLD, even after multiple-dosing regimens [38]. 


\section{Complement Activation-related Pseudoallergy (CARPA)}

Some liposomal structures are able to cause the innate immune reaction, with next activation of the complement system to spark an acute allergy syndrome called complement activation-related pseudoallergy (CARPA). The complement system is a part of the innate immune response, and is involved in a number of immunological and inflammatory strategies [39] A noticeably increased percent of victims (2-45\%) had been stated to broaden infusion-associated hypersensitive responses to liposomal drug delivery. In addition, CARPA has been stated with both experimental and clinically accepted liposomal phrasings (e.g., Doxil@, Ambisome, and DaunoXome®) [40]. CARPA is an immediate, nonIgE-mediated hypersensitivity response that involves signs and symptoms which include anaphylaxis, facial flushing, facial lump, headache, chills, and cardiopulmonary complications [41] the ultimate of which might also limit the medical use of doubtlessly reactogenic liposomes in cardiac cases [42] General scientific management includes decelerating the infusion rate or stoppage of delivery, as well as the use of allergic medications(e.g., antihistamines, epinephrine, corticosteroids).

\section{The enhanced Permeability and Retention (EPR) effect}

Liposomes which have escaped the RES and opsonization are carried to the EPR effect [43]. The EPR effect refers to the bettered permeability of the vasculature that give pathological tissues (e.g., tumors and conditions regarding inflammation). At these spot, deregulations in angiogenesis and/or the accelerated expression and activation of vascular permeability factors predominates [44] which leads to fenestrations which could variety from 300 to $4700 \mathrm{~nm}$. This lets in liposomes to extravasate and acquire via passive concentrated on [45]. All kinds of liposomal delivery structures are carried to the EPR impact, with PEGylated liposomes having an advantage due to having dropped RES clearance and prolonged circulate time [46].

\section{Experimental use of Liposomes for Biomedical packages}

The application of liposomes in medication offers enormous possibility for novel and effective remedies in an extensive variety of pathological situations. Because the discovery of liposomes over 50 years in the past, there was a sizable growth in lipid-based drug transport research on the experimental In vitro and In vivo section. Liposomes had been applied as a drug transport provider for a huge variety of therapeutic compounds and diagnostic agents which includes drug molecules, gene therapy and bioactive agents [47]. Modifications of those formulations are constantly being investigated if you want to improve efficacy, reduce RES clearance and limit toxicity - this consists of change in lipid composition charge, and the addition of surface coatings and ligands [48]. Recent strategies to improve on traditional or stealth liposomal structures involve active concentrated on, charged lipids, triggered release and multi-functional formulations [49].

\section{Targeted ligands}

Targeted ligands are used to ameliorate the specificity of transport of encapsulated shipment to and hold it in diseased tissues and cells, with minimum deposition in non-target sites. The notion that ligand-focused liposomes have a healing gain over non-targeted liposomes remains problem to discuss, with disagreeing consequences in the literature [50].Some of studies have verified more desirable uptake and efficacy of ligand-targeted liposomes in diseased tissue in evaluation to non-centered liposomes In vivo [51]. For instance, attachment of folate to liposomes showed better biodistribution of liposomes in folate-expressing tumors in a murine model [52].

\section{Charged lipids}

Adaptation of the lipid bilayer with charged lipids have additionally attracted lots of attention [53].Addition of charged lipids to the liposomal bilayer has performed a vital function in growing bioadhesive, mucoadhesive and nucleic acidbased delivery systems. As an instance, enhancing the surface charge of nano-shipping structures can have an effect on the electrostatic interplay of the nanocarriers with additives inside the gastrointestinal (GI) tract following oral management, and theoretically must confer selectivity to diseased tissue[54]. Cationic nano-shipping systems have been shown to adhere to the mucosal floor inside inflamed GI tissue, due to the interaction among the positively charged nanocarrier and the negatively charged intestinal mucosa [55]. Colonic mucins bring a negative charge since their carbohydrates are substituted with numerous sulfate and sialic acid residues [56]. Conversely, anionic nano-transport systems preferentially adhere to infected GI tissue via electrostatic interaction with the higher concentration of positively charged proteins in infected areas. Mainly, high amounts of eosinophil cationic protein and transferrin were determined in inflamed colon sections in patients with inflammatory bowel sickness (IBD) [57]. Cationic nano-delivery 
structures also are capable of successfully transport massive, charged systems,which includes DNA and RNA, based totally on electrostatic interplay among the positively charged phospholipids [e.g., dioleoylphosphatidylethanolamine (DOPE)] and negatively charged nucleic acids [58].

\section{Triggered release}

Another approach to improve therapeutic efficacy of liposomal formulations has been to use triggering modalities for site-specific release of therapeutics from liposomes [59]. Strategies that have been utilized include remote triggers (e.g., temperature, ultrasound, magnetic, and light) and local triggers specific to the target site (e.g., enzymes and pH), through the use of specific lipid compositions and coatings [60]. Of these strategies, the use of an external hyperthermic trigger to release therapeutic compounds from liposomal formulations (e.g., ThermoDox $囚$ ) appears to be the most promising to date [61]. Thermosensitive liposomes are modified with temperature-sensitive lipids (e.g., 1,2-distearoyl-sn-glycero3-phosphocholine, DSPC) and/or polymers [e.g., poly( $\mathrm{N}$-isopropylacrylamide)], which enables the nanocarrier to remain stable and retain their contents at physiologic temperatures.

\section{Challenges facing for delivery}

Challenges facing for Liposomal delivery have demonstrated significant remedial advantages for a multitude of biomedical operations, still the major reasons for the challenges have been attributed to issues girding therapeutical manufacturing, government regulations and intellectual property (IP). Analogous obstacles are faced by other nanodelivery systems for delivery into the clinic [62]. Limitations in pharmaceutical development are centered on quality assurance and cost. Quality assurance involves issues girding the manufacturing process and stability of the formulation, with nano-delivery systems being affected by (i) scalability of the manufacturing process, (ii) reliability and reproducibility of the final product, (iii) lack of equipment and/or in-house expertise, (iv) chemical instability or denaturation of the encapsulated compound in the manufacturing process, and (v) long term stability issues [63].Adding the number of physicochemical variables in a nanoformulation system also makes it more complicated to assess the pharmacokinetics, pharmacodynamics and toxicology of a formulation following administration [64]. For illustration, the use of synthetic coatings and ligands may affect the biocompatibility, biodistribution and toxicology profile of liposomal phrasings, and will need further evaluation to understand the interaction of the nanoparticles with biological tissues and cells [65].Eventually, clinical trials of liposomal phrasings are generally more complex than conventional phrasings, as a number of control groups are needed to regard for different aspects of the drug delivery system.

\section{Conclusion}

The use of liposomes to facilitate the administration of medicine has already had a major impact on many biomedical fields. Knowing the progress of liposomal technology so far and the challenges ahead, will enable future research to improve on existing platforms and to reflect the current limits in terms of translation and regulation. Ongoing translational success will demand communication and association between experts involved in all stages of therapeutic development of liposomal technologies, including manufacturing and therapeutic design, cellular interactions and toxicology, as well as preclinical and clinical evaluation.

\section{Compliance with ethical standards}

\section{Acknowledgments}

Sunil P M, Dean and Director, Stem cells and Regenerative medicine department, Sree Anjaneya Institute of Dental Sciences, Calicut, Kerala.

\section{Disclosure of conflict of interest}

No conflict of interest.

\section{References}

[1] Hemanthkumar M, Spandana V: Liposomal encapsulation technology a novel drug delivery system designed for ayurvedic drug preparation. IRJP. 2011; 2(10): 4-7. 
[2] Koning GA, Storm G. Targeted drug delivery systems for the intracellular delivery of macromolecular drugs. Drug Discov. Today. 2003; 8: 482-483.

[3] Hua S, Wu SY. The use of lipid-based nanocarriers for targeted pain therapies. Front. Pharmacol. $2013 ; 4: 143$.

[4] Ulrich AS. Biophysical aspects of using liposomes as delivery vehicles. Biosci. Rep. 2002; 22: 129-150.

[5] Koning GA, Storm G. Targeted drug delivery systems for the intracellular delivery of macromolecular drugs. Drug Discov. Today. 2003; 8: 482-483.

[6] Szebeni J, Moghimi SM. Liposome triggering of innate immune responses: a perspective on benefits and adverse reactions. J. Liposome Res. 2009; 19: 85-90.

[7] Gabizon et al. Koning and hurricane, 2003; Metselaar and hurricane, 2005; Ding et al., 2006; Hua and Wu. 2013. 1982.

[8] Gabizon AA, Barenholz Y, Bialer M. Prolongation of the circulation time of doxorubicin encapsulated in liposomes containing a polyethylene glycol-derivatized phospholipid: pharmacokinetic studies in rodents and dogs. Pharm. Res. 1993; 10: 703-708.

[9] Hua S, Wu SY. The use of lipid-based nanocarriers for targeted pain therapies. Front. Pharmacol. $2013 ; 4: 143$.

[10] Torchilin VP, Klibanov AL, Huang L, O'Donnell S, Nossiff ND, Khaw BA. Targeted accumulation of polyethylene glycol-coated immunoliposomes in infarcted rabbit myocardium. FASEB J. 1992; 6: 2716-2719.

[11] Gabizon AA, Barenholz Y, Bialer M. Prolongation of the circulation time of doxorubicin encapsulated in liposomes containing a polyethylene glycol-derivatized phospholipid: pharmacokinetic studies in rodents and dogs. Pharm. Res. 1993; 10: 703-708.

[12] Willis M, Forssen E. Ligand-targeted liposomes. Adv. Drug Deliv. Rev. 1998; 29: 249-271.

[13] Bendas G. Immunoliposomes: a promising approach to targeting cancer therapy. BioDrugs. 2001; 15: $215-224$.

[14] Ning YM, He K, Dagher R, Sridhara R, Farrell AT, Justice R, et al. Liposomal doxorubicin in combination with bortezomib for relapsed or refractory multiple myeloma. Oncology (Williston Park). 2007; 21: 1503-1508.

[15] Schmitt CJ, Dietrich S, Ho AD, Witzens-Harig M. Replacement of conventional doxorubicin by pegylated liposomal doxorubicin is a safe and effective alternative in the treatment of non-Hodgkin's lymphoma patients with cardiac risk factors. Ann. Hematol. 2012; 91: 391-397.

[16] Rahman AM, Yusuf SW, Ewer MS. Anthracycline-induced cardiotoxicity and the cardiac-sparing effect of liposomal formulation. Int. J. Nanomedicine. 2007; 2: 567-583.

[17] Chang TC, Shiah HS, Yang CH, Yeh KH, Cheng AL, Shen BN, et al. Phase I study of nanoliposomal irinotecan (PEP02) in advanced solid tumor patients. Cancer Chemother. Pharmacol. 2015; 75: 579-586.

[18] Zamboni WC, Ramalingam S, Friedland DM, Edwards RP, Stoller RG, Strychor S, et al. Phase I and pharmacokinetic study of pegylated liposomal CKD-602 in patients with advanced malignancies. Clin. Cancer Res. 2009; 15: 14661472.

[19] Willis M, Forssen E. Ligand-targeted liposomes. Adv. Drug Deliv. Rev. 1998; 29: 249-271.

[20] Sawant RR, Torchilin VP. Challenges in development of targeted liposomal therapeutics. AAPS J. 2012; 14: 303315.

[21] Poste G, Papahadjopoulos D, Vail WJ. Lipid vesicles as carriers for introducing biologically active materials into cells. Methods Cell Biol. 1976; 14: 33-71.

[22] Senior JH. Fate and behavior of liposomes In vivo: a review of controlling factors. Crit. Rev. Ther. Drug Carrier Syst. 1987; 3: 123-193.

[23] Sapra P, Allen TM. Ligand-targeted liposomal anticancer drugs. Prog. Lipid Res. 2003; 42: 439-462.

[24] Chrai SS, Murari R, Ahmad I. Liposomes (a review) part two: drug delivery systems. BioPharm. 2002; 17: 40-43.

[25] Oku N, Namba Y. Long-circulating liposomes. Crit. Rev. Ther. Drug Carrier Syst. 1994; 11: 231-270.

[26] Ishida T, Harashima H, Kiwada H. Interactions of liposomes with cells In vitro and In vivo: opsonins and receptors. Curr. Drug Metab. 2001a; 2: 397-409. 
[27] Cullis PR, Chonn A, Semple SC. Interactions of liposomes and lipid-based carrier systems with blood proteins: relation to clearance behaviour In vivo. Adv. Drug Deliv. Rev. 1998; 32: 3-17.

[28] Willis M, Forssen E. Ligand-targeted liposomes. Adv. Drug Deliv. Rev. 1998; 29: 249-271.

[29] Geng S, Yang B, Wang G, Qin G, Wada S, Wang JY. Two cholesterol derivative-based PEGylated liposomes as drug delivery system, study on pharmacokinetics and drug delivery to retina. Nanotechnology. 2014; 25: 275103.

[30] Dams ET, Laverman P, Oyen WJ, Storm G, Scherphof GL, van Der Meer JW, et al. Accelerated blood clearance and altered biodistribution of repeated injections of sterically stabilized liposomes. J. Pharmacol. Exp. Ther. 2000; 292: 1071-1079.

[31] Ishida T, Harada M, Wang XY, Ichihara M, Irimura K, Kiwada H. Accelerated blood clearance of PEGylated liposomes following preceding liposome injection: effects of lipid dose and PEG surface-density and chain length of the first-dose liposomes. J. Control. Release. 2005; 105: 305-317.

[32] Ishida T, Ichihara M, Wang X, Yamamoto K, Kimura J, Majima E, et al. Injection of PEGylated liposomes in rats elicits PEG-specific IgM, which is responsible for rapid elimination of a second dose of PEGylated liposomes. $J$. Control. Release. 2006b; 112: 15-25.

[33] Laverman P, Carstens MG, Storm G, Moghimi SM. Recognition and clearance of methoxypoly (ethyleneglycol) 2000-grafted liposomes by macrophages with enhanced phagocytic capacity. Implications in experimental and clinical oncology. Biochim. Biophys. Acta. 2001; 1526: 227-229.

[34] Moghimi SM, Hunter AC. Capture of stealth nanoparticles by the body's defences. Crit. Rev. Ther. Drug Carrier Syst. 2001; 18: 527-550.

[35] Szebeni J. Complement activation-related pseudoallergy: a new class of drug-induced acute immune toxicity. Toxicology. 2005; 216: 106-121.

[36] Szebeni J, Barenholz Y. "Adverse immune effects of liposomes: complement activation, immunogenicity and immune suppression," in Harnessing Biomaterials for Nanomedicine: Preparation, Toxicity and Applications, ed P. S. Publishing (Singapore: Pan Stanford Publishing). 2009; 1-19.

[37] Szebeni J, Fontana JL, Wassef NM, Mongan, PD, Morse DS, Dobbins DE, et al. Hemodynamic changes induced by liposomes and liposome-encapsulated hemoglobin in pigs: a model for pseudoallergic cardiopulmonary reactions to liposomes. Role of complement and inhibition by soluble CR1 and anti-C5a antibody. Circulation. 1999; 99: 2302-2309.

[38] Sawant RR, Torchilin VP. Challenges in development of targeted liposomal therapeutics. AAPS J. 2012; 14: 303315.

[39] Nehoff H, Parayath NN, Domanovitch L, Taurin S, Greish K. Nanomedicine for drug targeting: strategies beyond the enhanced permeability and retention effect. Int. J. Nanomedicine. 2014; 9: 2539-2555.

[40] Hashizume H, Baluk P, Morikawa S, McLean JW, Thurston G, Roberge S, et al. Openings between defective endothelial cells explain tumor vessel leakiness. Am. J. Pathol. 2000; 156: 1363-1380.

[41] Hua S, Wu SY. The use of lipid-based nanocarriers for targeted pain therapies. Front. Pharmacol. $2013 ; 4: 143$.

[42] Puri A, Loomis K, Smith B, Lee JH, Yavlovich A, Heldman E, et al. Lipid-based nanoparticles as pharmaceutical drug carriers: from concepts to clinic. Crit. Rev. Ther. Drug Carrier Syst. 2009; 26: 523-580.

[43] Ferrari M. Nanovector therapeutics. Curr. Opin. Chem. Biol. 2005; 9: 343-346.

[44] Vingerhoeds MH, Storm G, Crommelin DJ. Immunoliposomes In vivo. Immunomethods. 1994; 4: $259-272$.

[45] Gabizon A, Horowitz AT, Goren D, Tzemach D, Shmeeda H, Zalipsky S. In vivo fate of folate-targeted polyethyleneglycol liposomes in tumor-bearing mice. Clin. Cancer Res. 2003; 9: 6551-6559.

[46] Bozzuto G, Molinari A. Liposomes as nanomedical devices. Int. J. Nanomedicine. 2015; 10: 975-999.

[47] Hua S, Marks E, Schneider JJ, Keely S. Advances in oral nano-delivery systems for colon targeted drug delivery in inflammatory bowel disease: selective targeting to diseased versus healthy tissue. Nanomedicine. 2015; 11: 1117-1132.

[48] Coco R, Plapied L, Pourcelle V, Jérôme C, Brayden DJ, Schneider YJ, et al. Drug delivery to inflamed colon by nanoparticles: comparison of different strategies. Int. J. Pharm. 2013; 440: 3-12. 
[49] Larsson JM, Karlsson H, Sjövall H, Hansson GC. A complex, but uniform O-glycosylation of the human MUC2 mucin from colonic biopsies analyzed by nanoLC/MSn. Glycobiology. 2009; 19: 756-766.

[50] Carlson M, Raab Y, Peterson C, Hällgren R, Venge P. Increased intraluminal release of eosinophil granule proteins EPO, ECP, EPX, and cytokines in ulcerative colitis and proctitis in segmental perfusion. Am. J. Gastroenterol. 1999; 94: 1876-1883.

[51] Felgner PL, Gadek TR, Holm M, Roman R, Chan HW, Wenz M, et al. Lipofection: a highly efficient, lipid-mediated DNA-transfection procedure. Proc. Natl. Acad. Sci. U.S.A. 1987; 84: 7413-7417.

[52] Bibi S, Lattmann E, Mohammed AR, Perrie Y. Trigger release liposome systems: local and remote controlled delivery? J. Microencapsul. 2012; 29: 262-276.

[53] Guo X, Szoka FC Jr. Chemical approaches to triggerable lipid vesicles for drug and gene delivery. Acc. Chem. Res. 2003; 36: 335-341.

[54] Needham D, Anyarambhatla G, Kong G, Dewhirst MW. A new temperature-sensitive liposome for use with mild hyperthermia: characterization and testing in a human tumor xenograft model. Cancer Res. 2000; 60: 1197-1201.

[55] Allen TM, Cullis PR. Drug delivery systems: entering the mainstream. Science. 2004; 303: 1818-1822.

[56] Narang AS, Chang RK, Hussain MA. Pharmaceutical development and regulatory considerations for nanoparticles and nanoparticulate drug delivery systems. J. Pharm. Sci. 2013; 102: 3867-3882.

[57] Teli MK, Mutalik S, Rajanikant GK. Nanotechnology and nanomedicine: going small means aiming big. Curr. Pharm. Des. 2010; 16: 1882-1892.

[58] Allen TM, Cullis PR. Liposomal drug delivery systems: from concept to clinical applications. Adv. Drug Deliv. Rev. 2013; 65: 36-48. 